\title{
Application of integrative thermodynamic-hemodynamic- pharmacokinetic model to propofol anesthesia for hypothermic decompression
}

\author{
L. Gaohua \& H. Kimura \\ Bio-Mimetic Control Research Center, \\ The Institute of Physical and Chemical Research (RIKEN), Japan.
}

\begin{abstract}
A novel strategy of propofol anesthesia is proposed to improve clinical propofol anesthesia for hypothermic decompression. Theoretical analysis is carried out based on our previously developed integrative model of thermodynamics, hemodynamics and pharmacokinetics, after introducing a linear relationship of plasma propofol concentration with temperature thresholds of thermoregulatory responses. The therapeutic strategy proposed and simulated tentatively consists of four steps, that is, (i) the elevated intracranial pressure is decreased by therapeutic cooling; (ii) the brain temperature is cooled down simultaneously; (iii) the minimum plasma propofol concentration is calculated according to the propofol-threshold relationship and (iv) an adequate propofol is administered to realize the desired minimum plasma concentration. Simulation results suggest that the proposed propofol anesthesia is more appropriate than the empirical Roberts' step-down infusion schema for hypothermic decompression.

Keywords: brain hypothermia treatment, cooling, modeling, simulation, control.
\end{abstract}

\section{Introduction}

High intracranial pressure (ICP) is still a major cause of mortality in the intensive care unit [1]. Therefore, achieving a sustained reduction in ICP in patients with intracranial hypertension remains a great challenge in the clinical practice. Due to its hypothermic decompression, brain hypothermia treatment has been verified to be especially effective in such patients with refractory intracranial hypertension, in whom all other conventional therapeutic options 
have failed in decompression [2]. Actually, about half of brain hypothermia treatments were introduced for the purpose of control of refractory intracranial hypertension in German hospitals with neurosurgical care [3].

Adequate anesthesia, as well as effective management of intracranial temperature and pressure, is another important task in the therapeutic hypothermia. Propofol is widely used in the current clinical practice [4]. The rates of propofol administration are mainly based on the clinical experience and especially based on the normothermic dosage guideline. For example, an empirical administration known as the Roberts' step-down infusion scheme is used in neurosurgical patients undergoing general anesthesia.

However, the propofol kinetics in hypothermia is significantly different from that in normothermia, mainly because the enzymes that metabolize most drugs are extremely temperature-sensitive [5]. At the same time, the propofol kinetics is influenced indirectly by hypothermia as the hemodynamics is disturbed by therapeutic cooling. Therefore, a propofol administration strategy corresponding to the therapeutic cooling should be developed to improve the clinical propofol anesthesia in the hypothermic decompression.

The effects of hypothermia on propofol kinetics, on the one hand, have not been taken into account theoretically, although some physiologically based pharmacokinetic (PBPK) models for propofol have been developed recently $[6,7]$. On the other hand, some hints are available as an integrative model of thermodynamics, hemodynamics and pharmacokinetics of diuretic (mannitol) has been developed for patient under brain hypothermia treatment $[8,9]$.

The aim of this paper is to apply this integrative model to analyze propofol anesthesia for hypothermic decompression. Especially, a linear relationship between the temperature threshold (or in term of the triggering core temperature) of thermoregulatory response and the plasma propofol concentration is introduced to combine the thermodynamics and propofol kinetics together.

\section{Methods}

\subsection{Relationship of thermoregulatory threshold and plasma propofol concentration}

Concerning the dependence of thermoregulatory response thresholds on the plasma propofol concentrations, Matsukawa and colleagues [10] made a systemic investigation of thermoregulation under propofol anesthesia. They found that propofol increased the sweating threshold slightly, but it markedly reduced the vasoconstriction and shivering thresholds. Especially, propofol linearly decreased the core temperature triggering vasoconstriction or shivering.

Matsukawa's results could be described mathematically as follows,

$$
T_{\text {thres }}=T_{\text {othres }}-\sigma C_{\text {artery }}
$$

where $\mathrm{C}_{\text {artery }}\left(\mu \mathrm{g} \mathrm{ml}^{-1}\right)$ denotes the artery plasma concentration of propofol. $\sigma$ is the slope, $\sigma=0.6^{\circ} \mathrm{C}\left(\mu \mathrm{g} \mathrm{ml}^{-1}\right)^{-1}$ for vasoconstriction and $\sigma=0.7^{\circ} \mathrm{C}\left(\mu \mathrm{g} \mathrm{ml}^{-1}\right)^{-1}$ for shivering. $\mathrm{T}_{\text {thres }}\left({ }^{\circ} \mathrm{C}\right)$ is the temperature threshold of thermoregulatory response, 
and $\mathrm{T}_{0 \text { thres }}$ is its initial value. It was estimated that $\mathrm{T}_{0 \text { thres }}=36.5^{\circ} \mathrm{C}$ for vasoconstriction and $\mathrm{T}_{0 \text { thres }}=35.6^{\circ} \mathrm{C}$ for shivering [10].

The formula (1), named propofol-threshold mechanism hereafter, combines the thermodynamics with the pharmacokinetics of propofol algebraically. It also hints on how to anesthetize the patient undergoing hypothermic decompression to realize a stable management of physiological functions. Especially, the required plasma propofol concentration for hypothermic decompression could be calculated directly by using the formula (1), if considering the actual brain temperature of the patient is slight higher than the apparent temperature threshold determined by the plasma propofol concentration.

\subsection{Model description}

An integrative model of patient under brain hypothermia treatment has been modeled previously $[8,9]$. As shown in Fig. 1, the model consists of 6 segments or 13 lumped compartments. The cooling blanket is assumed to be applied to the mass compartment of muscular segment while the temperature and hydrostatic pressure in the cerebrospinal fluid (CSF) compartment are considered as the target brain temperature and ICP. Previously, the hypothermic effects on the hemodynamics and the pharmacokinetics of diuretic (mannitol) were considered to realize a simultaneous control of intracranial temperature and pressure [9].

In this paper, the thermodynamic and hemodynamic parts of the integrative model are used as they are, while the pharmacokinetic part of diuretic is revised to describe the propofol kinetics, mainly by changing the pharmacokinetic parameters of the model. Some assumptions are made for modeling the propofol kinetics. For example, propofol is administered by bolus injection or continuous infusion into the venous compartment, as shown in Fig. 1. The elimination of propofol occurs only in the visceral mass compartment.

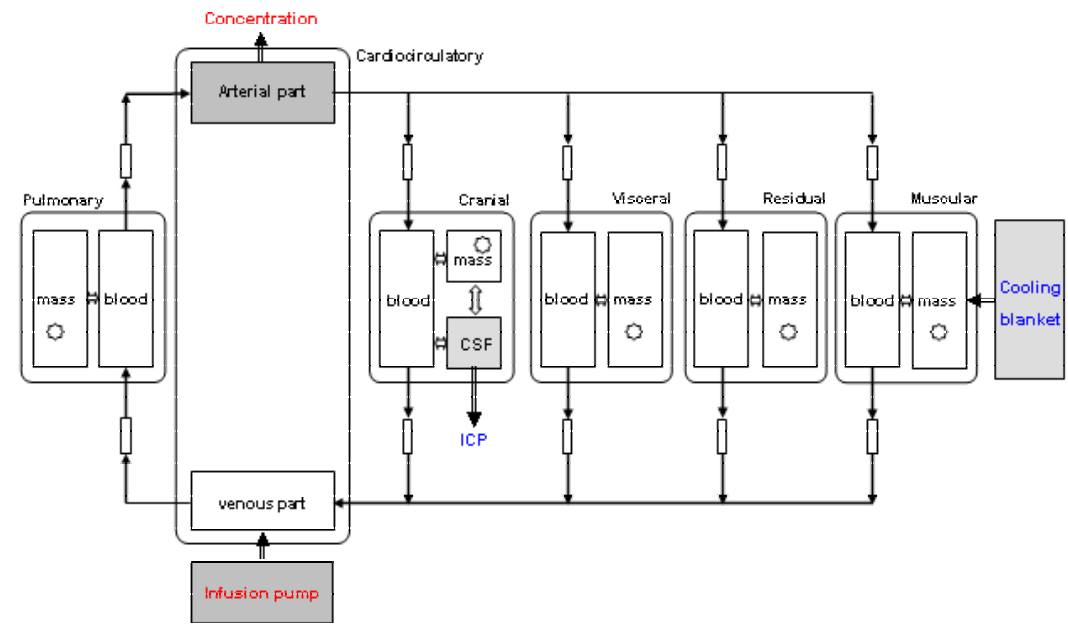

Figure 1: Compartmental structure of the integrative thermodynamichemodynamic-pharmacokinetic systems. 


\subsection{Governing equation}

All dynamics of temperature, hydrostatic pressure and propofol concentration in the compartmental model could be described mathematically based on the conservation laws of energy and/or mass [9]. For example, the propofol kinetics is described systemically by the following equation,

$$
\mathbf{V} \frac{d \mathbf{C}(t)}{d t}=\mathbf{A}(T, P, t) \mathbf{C}(t)+\mathbf{u}(t)
$$

where $\mathbf{V}$ is a diagonal matrix corresponding the distribution volume of propofol in each of the 13 compartments. $\mathbf{C}$ is state variable vector representing propofol concentration in each compartment, $\mathbf{A}$ is a time-varying coefficient matrix determined by both the pharmacokinetic parameters of propofol and the physiological states of the thermodynamics and hemodynamics. Interactions among the thermodynamics, the hemodynamics and the pharmacokinetics are involved in the coefficient matrix $\mathbf{A}$ through various temperature dependent mechanisms, as well as through blood flows. Therefore, matrix $\mathbf{A}$ is a function of temperature and pressure as well as time. The input vector $\mathbf{u}$ represents the propofol infusion into the venous compartment (Fig. 1).

\subsection{Pharmacokinetic parameters}

Physical and physiological parameters for the thermodynamics and hemodynamics are available in the previous studies $[8,9]$. Propofol permeability coefficient and total body clearance are given as follows.

a) Permeability coefficient Because propofol is highly protein bound (98-99\%) and only free or unbound propofol in the plasma could enter across the vascular wall, it is assumed that $1.5 \%$ of the apparent propofol concentration in the mass or blood compartment is concerned in the penetration across the vascular wall. The permeability coefficient of propofol across the blood-brain barrier is $0.511 \mathrm{~min}^{-1}$ [6]. The permeability coefficients of propofol are temperature dependent, which can be described by Arrhenius equation [8].

b) Total body clearance Since the total body clearance of propofol ranges from $23-50 \mathrm{ml} \mathrm{kg}{ }^{-1} \min ^{-1}[11,12]$, a middle value of $36.5 \mathrm{ml} \mathrm{kg}^{-1} \mathrm{~min}^{-1}$ is selected. As the enzymes that metabolize propofol are temperature-sensitive, the total body clearance is temperature dependent. The van't Hoff equation has been introduced to describe such temperature dependence of the clearance [8].

\subsection{Model verification}

As the thermodynamic and hemodynamic parts of the integrative model have been well validated previously $[8,9]$, only the pharmacokinetic part of the model is verified. Various propofol infusion rates are assumed to the pharmacokinetic model, and then the simulation results of transient behavior of the model are compared with the published clinical data or theoretical results.

Figure 2 shows an example of the agreement of the simulated propofol concentration in the arterial blood and that in the CSF with the data measured by Engdahl and colleagues [13], where a general anesthesia was induced into 
neurosurgical patients with propofol $2 \mathrm{mg} \mathrm{kg}^{-1}$ intravenously within $2 \mathrm{~min}$ and maintained with a continuous infusion of propofol commenced $5 \mathrm{~min}$ after the start of induction at an initial infusion rate of $8 \mathrm{mg} \mathrm{kg}^{-1} \mathrm{~h}^{-1}$ for $15 \mathrm{~min}$ and then reduced to $6 \mathrm{mg} \mathrm{kg}^{-1} \mathrm{~h}^{-1}$.

Concerning the simulation results, plasma propofol concentrations of the model at 2.5, 5, 15 and $30 \mathrm{~min}$ are 5.0, 1.8, 2.7 and $2.8 \mu \mathrm{g} \mathrm{ml}^{-1}$, respectively, as shown in Fig. 2(a). The plasma concentration of propofol increased rapidly during induction. After the bolus, the concentration decreased rapidly. It is consistent with the pharmacokinetics of propofol, that is, its rapid clearance from the blood produces the fast recovery characteristic of the drug.

As shown in Fig. 2(b), CSF propofol concentrations of the model always increased during the simulated duration. The concentrations at 2.5, 5, 15 and 30 min are 4.0, 11.0, 26.2 and $38.1 \mathrm{ng} \mathrm{ml}^{-1}$, respectively. The CSF concentration of propofol at $30 \mathrm{~min}$ was $1.4 \%$ of the plasma concentration. Altogether, it has been shown in the Fig. 2 that the CSF propofol concentration is positively correlated with and much lower than the plasma propofol concentration.

Both the predicted propofol concentration in the arterial blood and that in the CSF are of similar orders as the published data. Especially, in neurosurgical patients, the propofol concentration in CSF was 50- to 100-fold lower than in blood [13]. The simulation result (1.4\%) agrees well with the clinical knowledge. At this initial stage, the integrative model is considered to be applicable to theoretical analysis of propofol anesthesia, although further revisions would improve the model prediction.
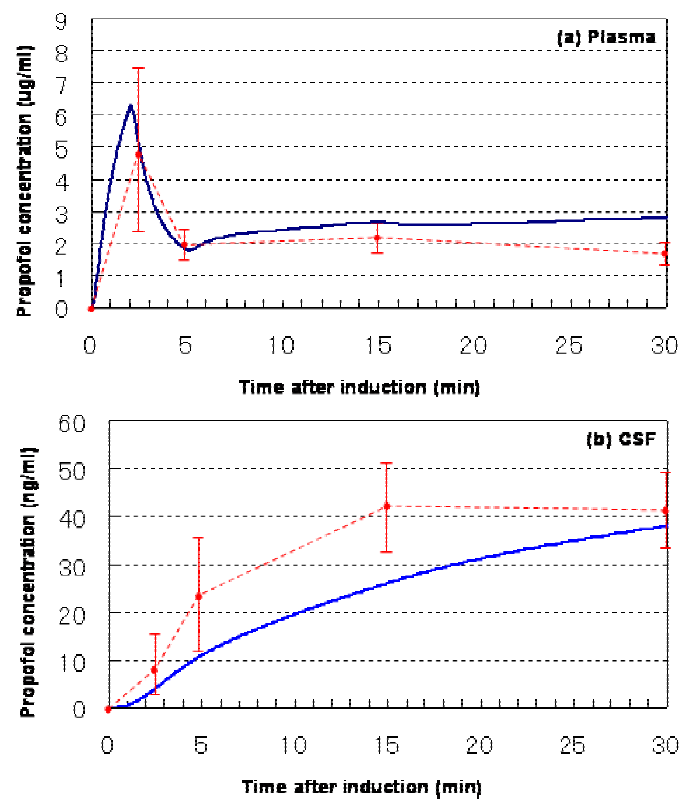

Figure 2: Propofol concentrations in plasma and CSF over time. (a) Plasma. (b) CSF (simulated data: solid line, reported data: dotted line). 


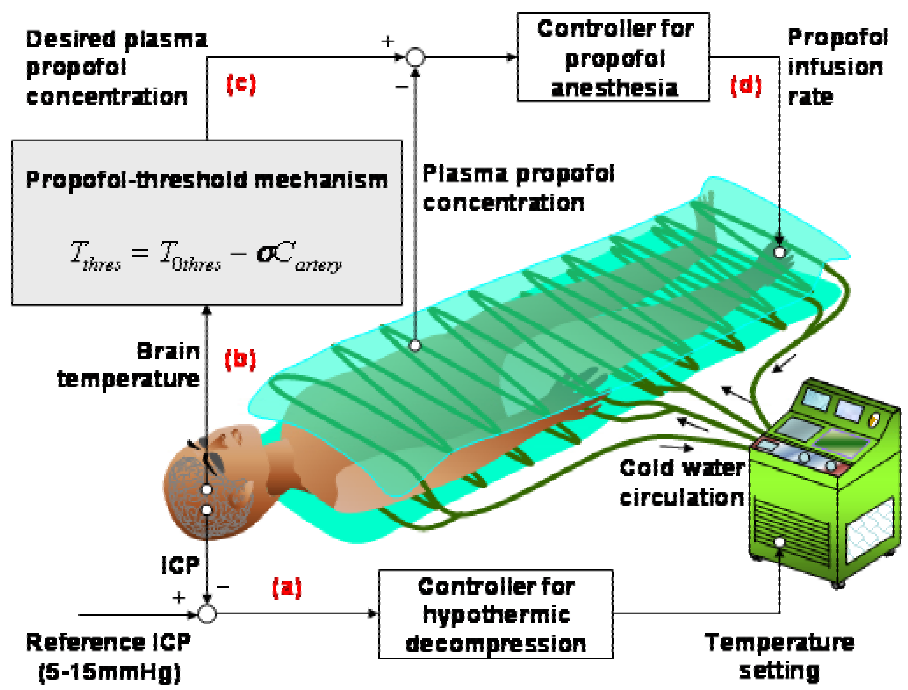

Figure 3: Therapeutic scenario of propofol anesthesia for hypothermic decompression.

\section{Application of model to propofol anesthesia}

\subsection{Therapeutic strategy of propofol anesthesia}

A therapeutic strategy of propofol anesthesia is proposed for hypothermic decompression. As shown in Fig. 3, it consists of 4 steps corresponding to a clinical scenario deciding an adequate propofol administration when brain hypothermia treatment is carried out to control the elevated ICP of the patient. The therapeutic strategy is simulated with the help of the integrative model and the propofol-threshold mechanism of formula (1), as follows,

(a) Hypothermic decompression The elevated ICP is decreased by inducing therapeutic cooling. Such a hypothermic decompression will be simulated in the hemodynamic part of the integrative model with a PID feedback temperature controller. For this purpose, a pathophysiological state of elevated ICP and a PID feedback temperature controller, both of which have been developed previously [9], are introduced.

(b) Brain temperature prediction Intracranial temperature is also cooled down by the therapeutic cooling induced for hypothermic decompression in Step (a). The CSF temperature (brain temperature) will be predicted by using the thermodynamic part of the integrative model.

(c) Concentration calculation The required minimum plasma concentration of propofol inhibiting the thermoregulatory responses is calculated mathematically by using the formula (1). The apparent temperature threshold determined by the plasma propofol concentration should be slightly below $\left(0.01^{\circ} \mathrm{C}\right.$ in the simulation) the cooled brain temperature predicted in Step (b). 
(d) Propofol administration An adequate titration of propofol administration is simulated by a PID feedback propofol controller to realize the required plasma concentration determined in Step (c). The pharmacokinetic part of the integrative model is introduced to the simulation and the PID feedback propofol controller is designed based on the dynamic response of the propofol kinetics with respect to a step-like propofol infusion.

Although the therapeutic scenario is divided into 4 steps in series, interactions among the thermodynamics, the hemodynamics and the pharmacokinetics occur simultaneously in the integrative model, as happened in a real patient during hypothermic decompression. The propofol-threshold mechanism of formula (1) with $\sigma=0.6^{\circ} \mathrm{C}\left(\mu \mathrm{g} \mathrm{ml}^{-1}\right)^{-1}$ and $T_{0 \text { thres }}=36.5^{\circ} \mathrm{C}$ is used since the plasma propofol concentration inhibiting the shivering is smaller than that for inhibition of vasoconstriction at a core temperature below $36.5^{\circ} \mathrm{C}$.

\subsection{PID propofol controller}

According to the step response of plasma propofol concentration to constant propofol infusion rate (data not shown), the dynamic response of plasma propofol concentration could be approximated by a linear transfer function,

$$
G(s)=\frac{k}{\left(1+\tau_{1} s\right)\left(1+\tau_{2} s\right)}
$$

where $\mathrm{G}$ denotes the transfer function and $\mathrm{s}$ is Laplace operator. $k$ and $\tau$ are static gain and time constant. They are estimated as follows, $k=0.63\left(\mu \mathrm{g} \mathrm{ml}^{-1}\right)\left(\mathrm{mg} \mathrm{kg}^{-1}\right.$ $\left.\mathrm{h}^{-1}\right)^{-1}, \tau_{1}=0.78 \mathrm{~h}, \tau_{2}=0.03 \mathrm{~h}$.

A PID feedback controller is developed to tune the propofol infusion rate to realize a required plasma propofol concentration,

$$
\begin{gathered}
I(t)=K^{P}\left(e(t)+\frac{1}{K^{I}} \int_{0}^{t} e(\tau) d t+K_{D} \frac{d e(t)}{d t}\right) \\
K^{P}=\frac{\tau_{1}+\tau_{2}}{\lambda k}, K^{I}=\tau_{1}+\tau_{2}, K^{D}=\frac{\tau_{1} \tau_{2}}{\tau_{1}+\tau_{2}}
\end{gathered}
$$

where I $\left(\mathrm{mg} \mathrm{kg}^{-1} \mathrm{~h}^{-1}\right)$ is the infusion rate of propofol. e $\left(\mu \mathrm{g} \mathrm{ml}^{-1}\right)$ is the controlled error of plasma propofol concentration, $\lambda=0.8 \mathrm{~h}$.

\section{Simulation results and discussion}

The proposed propofol anesthesia is simulated. The reference ICP is $15 \mathrm{mmHg}$. For comparison, the empirical schema of Roberts' step-down propofol infusion, that is, $10 \mathrm{mg} \mathrm{kg}^{-1} \mathrm{~h}^{-1}$ (0-10 min), $8 \mathrm{mg} \mathrm{kg}^{-1} \mathrm{~h}^{-1}$ (10-20 min), $6 \mathrm{mg} \mathrm{kg}^{-1} \mathrm{~h}^{-1}(20-30$ $\mathrm{min}$ ) and $5 \mathrm{mg} \mathrm{kg}^{-1} \mathrm{~h}^{-1}$ (30 min - the end of simulation), is simulated too.

Simulation results are shown in Fig. 4. Therefore,

(a) as shown in Fig. 4(a), the elevated ICP more than $24 \mathrm{mmHg}$ could be decreased to and kept around the reference ICP of $15 \mathrm{mmHg}$ in about $4 \mathrm{~h}$ after inducing the therapeutic cooling. The maximum speed of ICP decrease is 

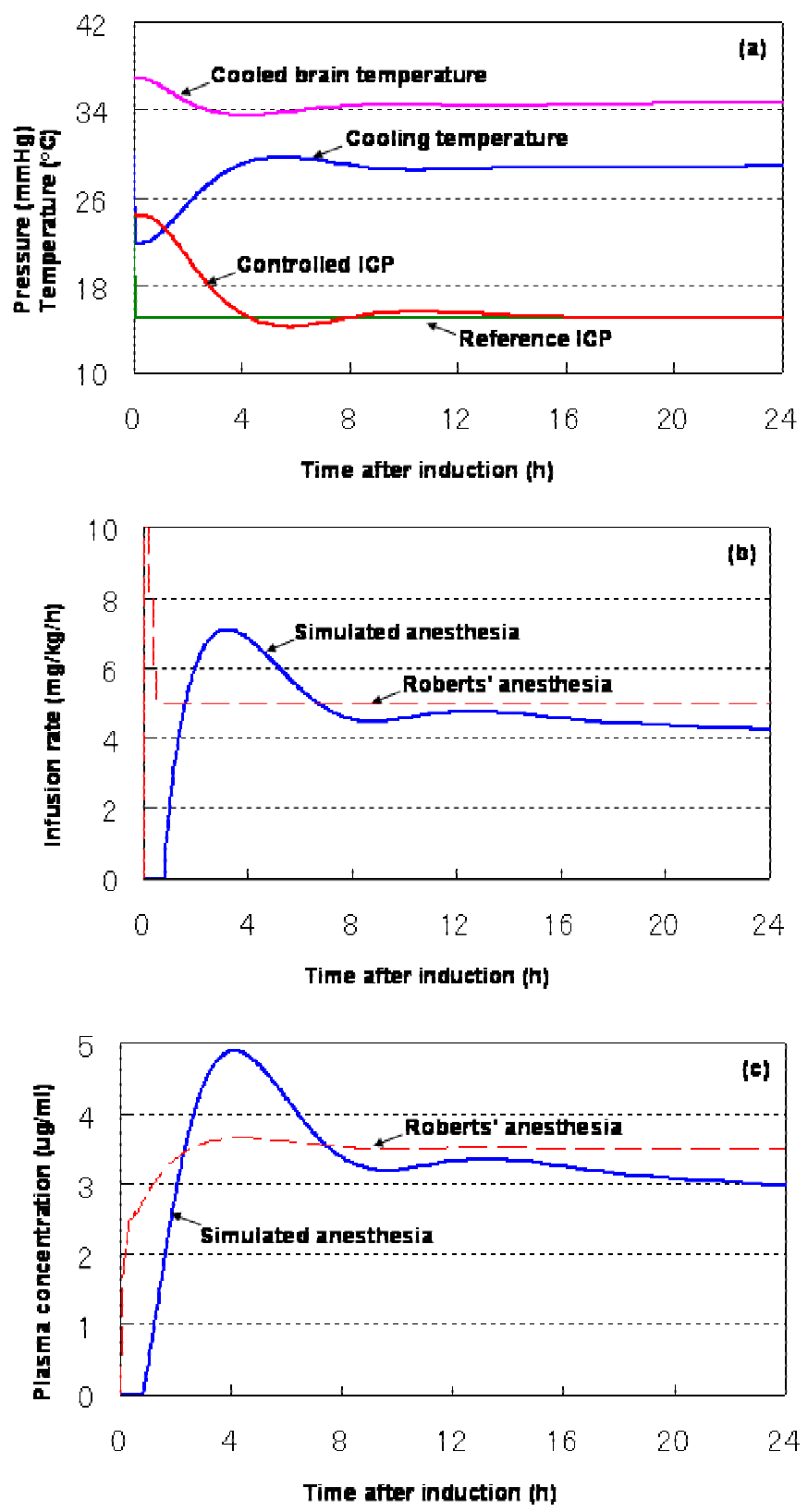

Figure 4: Simultaneous management of the elevated intracranial pressure, temperature and propofol anesthesia. (a) Hypothermic decompression. (b) Plasma concentration. (c) Infusion rate (simulated anesthesia: solid line; Roberts' anesthesia: dotted line). 
$3.4 \mathrm{mmHg} \mathrm{h}^{-1}$ at about $2 \mathrm{~h}$. The overshoot of the controlled ICP is little than $5 \%$ $(0.74 \mathrm{mmHg})$, while the static error at the end of the simulation is little than 0.1 $\mathrm{mmHg}$. It reflects the effectiveness of hypothermic decompression.

(b) with the PID feedback temperature controller, the cooling temperature for decompression, which is also shown in Fig. $4(\mathrm{a})$, is about $22^{\circ} \mathrm{C}$ initially and then increases when ICP decreasing. With therapeutic cooling, the brain temperature predicted by the thermodynamic part is cooled down too. The static gain of the cooled brain temperature with respect to the cooling temperature is about $2.0^{\circ} \mathrm{C}$ ${ }^{\circ} \mathrm{C}^{-1}$, as their values at the end of simulation are $28.9^{\circ} \mathrm{C}$ and $34.7^{\circ} \mathrm{C}$ respectively. Such a dynamic regulation of cooling temperature is clinically practicable.

(c) as shown in Fig. 4(b), the minimum plasma concentration of propofol could be calculated according to the formula (1) while considering the apparent threshold of thermoregulation determined by the plasma concentration is $0.01^{\circ} \mathrm{C}$ below the brain temperature predicted in Step (b). The desired minimum plasma concentration was achieved by the PID feedback propofol controller.

Compared with the Roberts' propofol anesthesia, the plasma concentration of propofol is higher during the period of 2.3-7.5 h. It corresponds with the fact that the controlled brain temperature is low during this period. Quantitatively, the peak plasma concentration is $4.9 \mu \mathrm{g} \mathrm{ml}^{-1}$ at $4.1 \mathrm{~h}$, where the cooled brain temperature is about $33.6^{\circ} \mathrm{C}$. It is reasonable to suppose that the Roberts' anesthesia would fail to inhibit the thermoregulatory responses at this controlled brain temperature if no additional administration was titrated during this period.

(d) as shown in Fig. 4(c), the simulated propofol administration varies dynamically according to the cooling temperature. Especially, it is during the first $0.8 \mathrm{~h}$ that no anesthesia is necessary, although the cooling temperature is most low. The reason is that the brain temperature is still higher than the thermoregulatory thresholds during this initial period, as shown in Fig. 4(a). By contrast, the infusion rate is high in the first half of an hour in Roberts' schema. It is also shown in Fig. 4(c) that the maximum infusion rate is $7.1 \mathrm{mg} \mathrm{kg}^{-1} \mathrm{~h}^{-1}$ at $3.2 \mathrm{~h}$, which is about $0.9 \mathrm{~h}$ before the occurrence of peak plasma concentration.

Compared with the empirical Roberts' schema, the total dosage of proposed anesthesia decreases more than $7 \%$ (total dosage is $121.5 \mathrm{mg} \mathrm{kg}^{-1}$ in Roberts' anesthesia while $113.0 \mathrm{mg} \mathrm{kg}^{-1}$ in the proposed anesthesia) during the simulated period of 24 hours. As pointed by McKeage and Perry [11], a higher than necessary dosage will lead to high plasma concentrations of propofol, which can result in a longer time to recovery. Thence the propofol administration suggested by the PID feedback propofol controller is clinically more practicable than the Roberts' step down infusion schema.

\section{Conclusion}

The main purpose of this paper was to apply the previously developed integrative model of thermodynamic-hemodynamic-pharmacokinetic systems to analyse the propofol anesthesia for hypothermic decompression. A linear relationship of the plasma propofol concentration with the temperature threshold of thermoregulatory response was introduced to combine the thermodynamics with the propofol kinetics algebraically. A therapeutic strategy of propofol 
anesthesia was proposed and simulated tentatively by using the integrative model. It was shown that the proposed propofol anesthesia is more appropriate than the empirical Roberts' step-down infusion schema. We suggest further in situ studies of the proposed anesthesia strategy to provide direct evidence to the clinical propofol anesthesia for hypothermic decompression.

\section{References}

[1] Georgiadis, A.L. \& Suarez, J.I., Hypertonic saline for cerebral edema. Curr. Neurol. Neurosci. Rep. 3, pp. 524-530, 2003.

[2] Polderman, K.H., Tjong, Tjin, Joe, R., Peerdeman, S.M., Vandertop, W.P. \& Girbes, A.R., Effects of therapeutic hypothermia on intracranial pressure and outcome in patients with severe head injury. Intensive Care Med. 28, pp. 1563-1573, 2002.

[3] Himmelseher, S. \& Werner, C., Therapeutic hypothermia after traumatic brain injury or subarachnoid hemorrhage. Current practices of German anaesthesia departments in intensive care. Anaesthesist 53, pp. 1168-1176, 2004.

[4] Hayashi, N. \& Dietrich, D.W., Brain hypothermia treatment. SpringerVerlag: Tokyo, pp. 1-325, 2004.

[5] Polderman, K.H., Application of therapeutic hypothermia in the intensive care unit. Opportunities and pitfalls of a promising treatment modalityPart 2: Practical aspects and side effects. Intensive Care Med. 30, pp. 757769, 2004.

[6] Upton, R.N. \& Ludbrook, G.L., A physiologically based, recirculatory model of the kinetics and dynamics of propofol in man. Anesthesiology 103, pp. 344-352, 2005.

[7] Levitt, D.G. \& Schnider, T.W., Human physiologically based pharmacokinetic model for propofol. BMC Anesthesiol. 5, 4. 2005.

[8] Gaohua, L. \& Kimura, H., A mathematical model of intracranial pressure dynamics for brain hypothermia treatment. J. Theor. Biol. 238, pp. 882900, 2006.

[9] Gaohua, L., Maekawa, T. \& Kimura, H., An integrated model of thermodynamic-hemodynamic- pharmacokinetic system and its application on decoupling control of intracranial temperature and pressure in brain hypothermia treatment. J. Theor. Biol. 242, pp. 16-31, 2006.

[10] Matsukawa, T., Kurz, A., Sessler, D.I., Bjorksten, A.R., Merrifield, B. \& Cheng, C., Propofol linearly reduces the vasoconstriction and shivering thresholds. Anesthesiology 82, pp. 1169-1180, 1995.

[11] McKeage, K. \& Perry, C.M., Propofol: a review of its use in intensive care sedation of adults. CNS Drugs 17, pp. 235-272, 2003.

[12] Product Information, Diprivan ${ }^{\circledR}$, propofol. AstraZeneca, Wilmington DE, http://www.astrazeneca-us.com/pi/diprivan.pdf

[13] Engdahl, O., Abrahams, M., Bjornsson, A., Vegfors, M., Norlander, B., Ahlner, J. \& Eintrei, C., Cerebrospinal fluid concentrations of propofol during anaesthesia in humans. Br. J. Anaesth. 81, pp. 957-959, 1998. 